\title{
EL FRUTO DE LA TECNOLOGÍA - DEBEMOS NOSOTROS ALIMENTAR UN PACIENTE ANCIANO A TODO COSTO?
}

Nili Tabak*

TABAK, N. El fruto de la tecnología - debemos nosotros alimentar un paciente anciano a todo costo?. Rev.latinoam.enfermagem, Ribeirão Preto, v. 5, n. 2, p. 61-67, abril 1997.

El próposito de este artículo es analizar las preguntas que rodean un común pero complejo dilema ético, médico y asistencial, llamado la justificación de continuar o suspender la alimentación forzada en pacientes extremadamente ancianos que sufren de aguda demencia progresiva. Tradicionalmente el principio de santidad de la vida apoya la idea que la vida debe ser prolongada a todo costo y la decisión de continuar com la alimentación, no se pone en duda. Sin embargo, últimamente han surgido gran cantidad de ideas revolucionarias hacia las actitudes, las concepciones y los valores del tratamiento, los cuales han guiado hacia una urgente necesidad de discutir el problema en un sentido mas profundo. Hasta qué punto debemos alimentar a la fuerza un paciente demente, el cual se niega a comer (sonda, alimentación intravenosa), atando sus manos o utilizando otras medidas? Esto ocurrío en un instituto geriátrico en Israel, donde una orden médica de un determinado médico consterno a las enfermeras de planta, las cuales decidieron que en este específico caso su punto de vista no era similar al del médico. El resultado fue una conferencia nacional con la participación de médicos, enfermeras, trabajadores sociales y sicólogos.

TÉRMINOS CLAVES: anciano, alimentación forzada, enfermera geriátrica

\section{INTRODUCCIÓN}

La expectativa por una larga vida ha creado muchos dilemas complejos, médicos y éticos, en el caso de pacientes ancianos.

El desarrollo tecnológico ha tenido éxito en prolongar la duración de la vida. Sin embargo, no siempre ha prosperado en cuanto a mejorar su calidad se refiere.

Hoy en día, la esperanza de vida esta alrededor de los 75 años, tanto para hombres como para mujeres, consecuentemente muchos de los dilemas médicos conciernen esta edad pactada.

En cierta forma, la acumulación de enfermedades y de deshabilidades conllevan a un deteríoro en el funcionamiento y como consecuencia de este mal funcionamiento a una pérdida de la independencia, un daño de la propia imagen y del estado mental del paciente. En estos casos, muchas veces el personal que proporciona el tratamiento está expuesto a serios conflictos, entre la obligación de asegurarse de proporcionar la mejor calidad de tratamiento al paciente anciano, y su obligación ante la sociedad. Esta última obligación afirma que el tratamiento no debe convertirse en un peso económico para los recursos de la sociedad, una carga que la sociedad no puede darse el lujo. Además estamos obligados a no prolongar el tiempo de vida si esto conlleva a un sufrimiento innecesario.
La imagen negativa de los ancianos en la sociedad moderna ha creado varios estereotipos de degeneración total de las destrezas y habilidades físicas, mentales y sociales, causando un completo desapego (GOMPERT, 1971; HAZAN, 1984; MOTALIS, 1989) ${ }^{1,2,3}$. El anciano es concebido en la sociedad como un participante desequivalente y una debida oportunidad para adaptarse a sus habilidades no le es concedida. El personal asistencial se encuentra atrapado en un conflicto de lealtad entre la necesidad de ser consecuente con los valores inculcados y entre las diferentes expectativas hacia el tratamiento por parte de los pacientes y sus familias.

Problemas de alimentación de pacientes ancianos que sufren de demencia senil son una realidad diaria, lo cual causa un gran sufrimiento tanto al personal asistencial como a los familiares. El sistema médico en Israel, al proporcionar tratamiento médico, no diferencia entre pacientes con gran potencial de rehabilitación y entre pacientes para los cuales sobrevivir una hora mas de vida es todo un éxito. Cada día somos testigos del hecho que tratamientos médicos y asistenciales son proporcionados inclusive en casos en los cuales el sistema no tiene la posibilidad de proveer tratamientos "prometedores".

En los últimos años, los pacientes están reaccionando en contra del paternalismo médico y están reclamando sus derechos de ser participantes activos en

* Departamento de Enfermería. Facultad de Medicina. Universidad de Tel Aviv 
el proceso de toma de decisiones concernientes a sus cuerpos y su derecho de rehusar tratamientos en vano. Obviamente, esta posición proviene de la teoría que sugiere que la vida mantenida en vano sin aparente calidad, no es deseada.

Este conflicto ha creado muchos serios dilemas, los cuales han sido complejamente analizados en debates a nivel médico, social y moral. Yo no se, si una respuesta absoluta puede darse inmediatamente. Sin embargo, debemos escoger entre las diferentes opciones, eligiendo un determinado modo de acción, el cual nos guiará al resultado deseado. Una enfermera geriátrica profesional debe saber como combinar los elementos humanos con los elementos emocionales, que la ayudarán a diagnosticar correctamente, evaluar apropiadamente y a planear efectivamente los resultados.

\section{Que es inutilidad médica?}

El concepto de inutilidad médica fue definido, en un principio en la literatura norteamericana, como un proceso cognitivo que justifica la prevención de tratamiento médico.

En realidad, el médico tiene el derecho de declarar (por supuesto basándose en consideraciones clínicas) que son apoyadas por el comité ético del hospital.

Sin embargo, otras importantes preguntas salen aquí a luz: ¿ Quién está autorizado a tomar decisiones sobre la útilidad de un tratamiento? ¿ Es el paciente, su familia, el personal médico, el personal asistente, el comité ético, la junta directiva del hospital - o tal vez un tribunal de justicia?

Estas preguntas son muy difíciles de responder, especialmente cuando el paciente está confuso y deseconectado de la realidad, no es un compañero activo en el proceso de toma de decisiones y está representado por su familia. Además se sugiere que las consideraciones económicas sobre el costo del tratamiento tienen una crucial influencia sobre la toma de dichas decisiones en el futuro.

Sin embargo, como sociedad, estamos obligados a tomar todas las medidas necesarias para preservar, como un valor moral sagrado y absoluto, la vida del ser humano. El no respetar este valor nos conducirá a un sendero en declive, cuyo final es incierto. Los derechos del ser humano de existir y de vivir están derivados de uno de los más importantes principios morales de la Biblia: “...No matarás...", por lo cual no nos está permitido poner fin a ninguna clase de vida humana.

Por un lado el médico desempeña el papel de agente de control social y por el otro lado es el representante del paciente. Estos dos papeles plantean un serio problema de doble lealtad, en el cual en algunas oportunidades la decisión de suspender un tratamiento es tomada a consecuencia de consideraciones institucionales o económicas.

Uno de los más grandes peligros será expuesto al capacitar a los profesionales de salud a decidir si el tratamiento médico es en vano o no. Estas decisiones, de gran peso moral, deben ser discutidas en varios paises. Las autoridades de estos paises deberán capacitar a las instituciones sanitarias como actuar a nivel microcósmico.

\section{ESTUDIO DE UN CASO}

El Sr. S.Z de tamaño medio, nació en Israel. Tenía 84 años. Fue hospitalizado en una institución geriátrica en muy malas condiciones físicas. Llegó a la institución acompañado de su familia, su esposa de 77 años de edad y de sus dos hijos de 49 y 50 años. El Sr. S.Z. estaba muy confuso la mayoría del tiempo, murmuraba mucho e parecía desconectado de la realidad. Fue diagnosticado profesionalmente como un anciano que necesita completo tratamiento asistencial. De acuerdo con su familia, S.Z. no estaba funcionando durante el último año y medio, perdió peso, se rehusada a comer y estaba confuso la mayor parte del tiempo.

El diagnóstico? O.M.C. - Demencia Senil Progresiva - Enfermedad tipo Alzheimer.

El paciente no controlaba sus esfínteres, vomitaba la comida, se volviá violento y cerraba sus mandíbulas. A la hora de la comida, el personal se veia forzado a atar el cuerpo del paciente y sus manos y debian alimentarlo a pesar de su gran resistencia. Esta situación causaba un gran sufrimiento al paciente y una gran tensión a las enfermeras. Cuando el doctor lo ordenó, una sonda le fue insertada y a ratos removida. La familia se resintió de este procedimiento argumentando que se le estaba causando un gran sufrimiento físico al paciente, además de ser estéticamente fastidioso. El personal profesional sabía que sí el paciente no era alimentado usando la sonda, su condición física se deteoraria y su vida estaría en peligro.

Los argumentos de la familia convencieron al doctor de la institución, el cual decidió suspender el tratamiento y no usar más el método de alimentación forzada. Las enfermeras de planta no estuvieron de acuerdo con la opinión del doctor y apelaron ante una autoridad más alta. Esta autoridad ordenó insertar la sonda nuevamente y de atar las manos del paciente. El paciente falleción una semana después.

A consecuencia que dentro del próprio instituto no se llegó a un concenso sobre como tratar el caso, la discrepancia codujó a una conferencia conjunta para los doctores y las enfermeras, en la cual los asuntos pudieran ser discutidos abiertamente. 
CONSIDERACIONES QUE INFLUENCIAN EL TRATAMIENTO

Las actitudes del personal médico y asistencial hacia el tratamiento de un paciente anciano se derivan de:

01. El concepto de la vida y el de ser anciano, desde un punto de vita social y cultural.

02. Definiciones de calidad de vida y los medios de mejorarla.

03. Los conceptos relativos a los derechos del paciente. 04. Puntos de vista sobre el tema de prioridades y recusos. 05. Principios concernientes a las actitudes hacia los ancianos.

06. Política local e institucional.

En una condición en la cual el paciente se niega a comer o que lo alimenten, la enfermera se encuentra en una situación de valores conflictivos.

El deber profesional de la enfermera es tratar el paciente, salvar su vida, conservar su dignidad y sus derechos - son todos estos valores internos de la conciencia; estos valores universales se rebelan ante del humillante sufrimiento causado por el método de alimentación forzada.

El básico conflicto moral del derecho de vivir a todo costo proviene del principio que predica que la vida es santa y el valor depreservarla tiene un mayor peso que el principio de la calidad de la vida. En este caso, el paciente reclama que su vida no tiene ninguna calidad. (SCHOSTAK, 1994) ${ }^{4}$.

(JACOBOVITCH, 1989) ${ }^{5}$ Hoy en día, como miembros del personal de tratamiento, estamos cada vez mas atentos ante el hecho que los conceptos universales y los diferentes puntos de vista han cambiado con los avances de la tecnología. Como consecuencia, muchas familias de enfermos perciben que el concepto de la calidad de vida y la prevención de sufrimiento tienen un mayor valor que preservación de la vida a todo costo. En tal caso, las limitaciones de tratamiento son frecuentemente producto de las opiniones, las creencias y los valores del médico, los cuales son consecuencia de la conciencia, la moral, la religión y las normas legales, sociales y económicos. (ZONGEBLIC, 1989) ${ }^{6}$. Debido al hecho que el deber de salvar vida esta muy enfatizado en la "Torá" y en el "Talmud", la gente que esta a cargo de proveer tratamiento médico, lo realiza de tal forma como si hubieran recibido instrucción moral y legal. Uno de los componentes de la habilidad de la persona para salvar vida es su destreza; por lo tanto, estamos demandando poner toda clase de esfuerzos personales y profesionales en orden de salvar la vida. (PROSCHT, 1992) ${ }^{7}$. Cuando el paciente se niega a comer, debido a su impedimiento mental, podemos ignorar su "opinión”, forzarlo a comer y salvarle la vida.

En Israel, la actitud mas común, es la de alimentar a todo costo y prolongar la vida, sin reparar en la calidad. La intervención de alimentación forzada está basada en la necesidad, que demanda el bién público, de salvar la vida inclusive contradiciendo otros intereses. Nosotros entendemos que esto conlleva un conflicto de intereses entre las demandas éticas y profesional del personal asistencial. Por outro lado, el personal asistencial debe estar en una posición donde alternativas pueden ser dadas.

Como modo de vida, el principio que guía a las enfermeras en Israel es el de prevenir sufrimiento; sin embargo, en la realidad muchas de ellas usan el método de alimentación forzada. (NORBERG \& HIRSCHFELD, $1987)^{8}$. El proceso de tomar decisiones por parte del personal asistencial afirma que el personal sabe que es lo mejor, como tratamiento, para el paciente. ¿. De esta manera debe ser?

Cuando recurrimos a la literatura, podemos encontrar diferentes actitudes hacia este asunto. Actitud 1 - concibe la alimentación como un estricto tratamiento médico. (DRESSER, 1985) ${ }^{9}$; mientras actitud 2 - concibe la alimentación como una necesidad básica para la existencia y supervivencia (LYNN et al.) ${ }^{10}$. Sea cual sea la actitud del personal asistencial, los sentimientos de impotencia que lo acompañan al proporcionar el tratamiento, son iguales (asi sea tratamiento forzado o no).

De éste modelo, podemos aprender que existen muchos métodos de alimentación a pacientes, que se rehusan a que se les alimente. Cada método es diseñado de acuerdo con una determinada política, derivada de los valores y las creencias de los miembros del personal, en el determinado instituto.

El proceso de toma decisiones debe realizarse de forma individual y cuando un método convencional falla debemos considerar el uso de medios de alimentación forzada.

Sin embargo, estos medios también crean una problemática médica y moral causando muchos conflictos y reflexiones entre el personal, tales como: “ $¿$ Debemos salvar la vida a todo costo, inclusive cuando es evidente el sufrimiento, la humillación y la degradación en esta particular situación?".

La correlación entre el deseo de alimentar y el código de principios éticos fue comprobado en una investigación realizada en varios países alrededor del mundo (NORBERG et al., 1994) ${ }^{11}$. Enfermeras con tendencia a alimentar a sus pacientes de vez en cuando, puntuaron altamente el principio ético de la santidad de la vida. Las enfermeras qu escogieron no alimentar a los pacientes ancianos dementes otorgaron el puntaje alto al principio ético de la autonomía. Muchas enfermeras atacaron el principio ético de utilitarianismo. Una de las principales preguntas concernientes a este hecho es: "i Qué clase de calidad de vida estamos proporcionando al usar métodos de alimentación forzada?"(MILMOE \& CARRICK, 1986) ${ }^{12}$. 

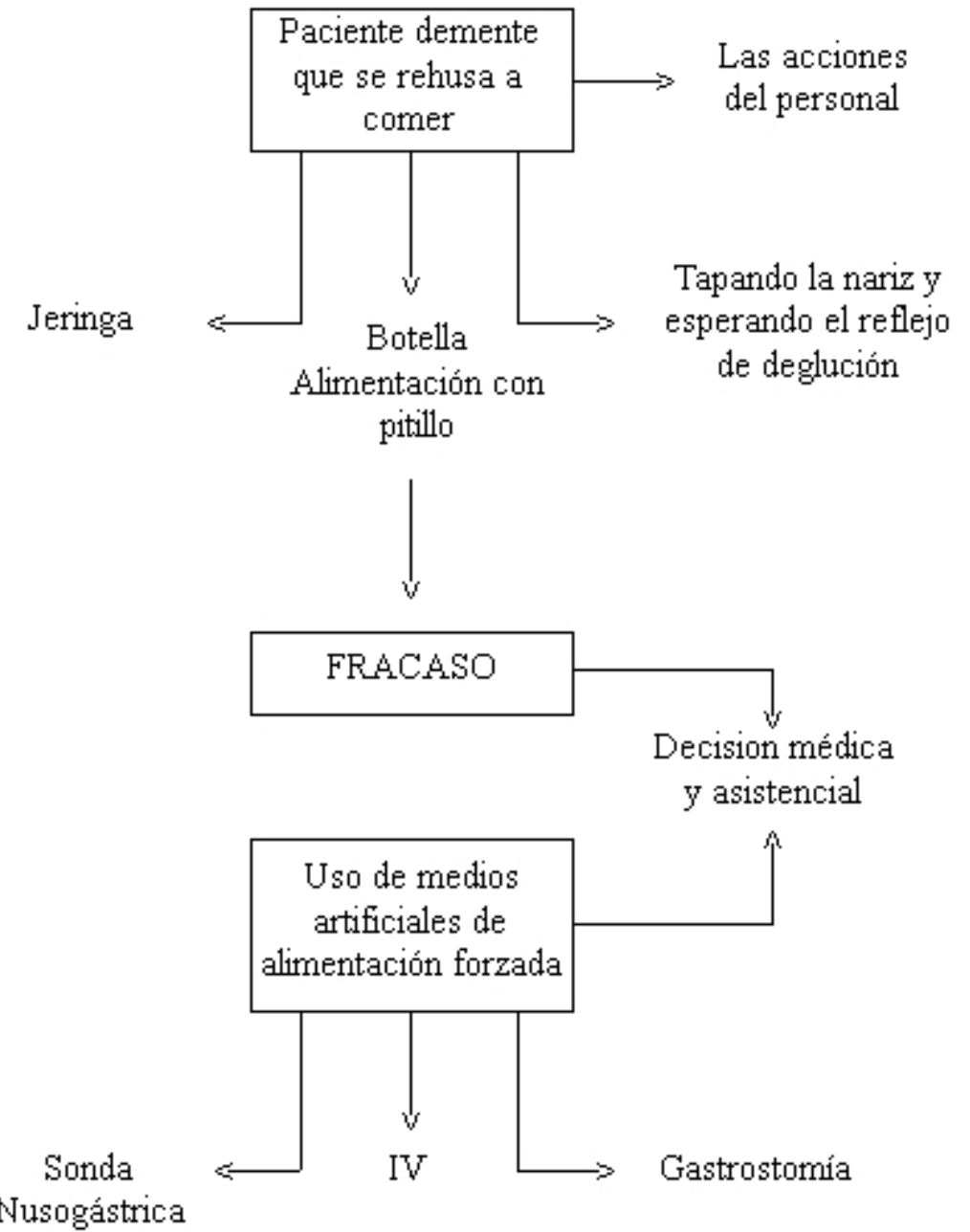

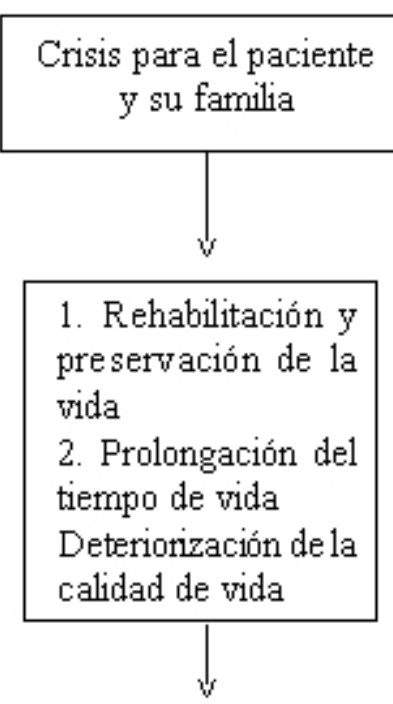

Debilidad

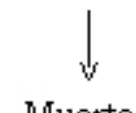

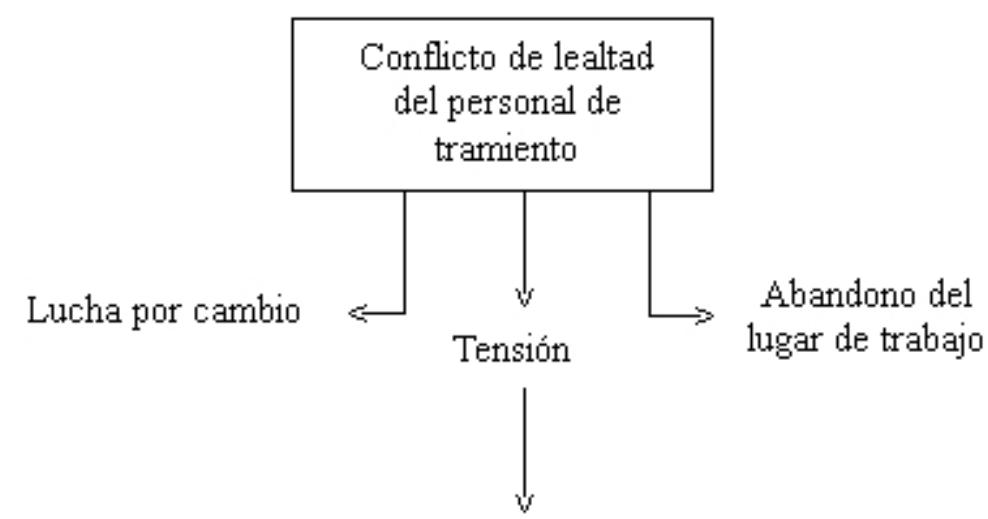

Frustración

tipo de vida

tiempo de vida

(Tabak, 1994) 
ANÁLISIS DE UN CASO DE ACUERDO CON LAS TEORÍAS FILOSÓFICAS

\section{Teoría teleológica}

Teoría que solamente toma en cuenta los resultados; lo cual significa, el personal está obligado a examinar cada opción, tomando en cuenta todas las consecuencias. Si se llega a la conclusión que cualquier clase de acción relacionada con el tratamiento puede causar sufrimiento, daño o inconveniencia, entonces se preferirá aplicar la doctrina de la ley natural - la prevención de alimentación forzada, y la terminación de la vida preservando la dignidad del paciente.

\section{Teoría deontológica}

La ejecución de una acción debe ser guiada por el principio y no por las consecuencias o los resultados de dicha acción. Preservación del principio de santidad de la vida humana guiará al personal a tratar de cualquier forma y a cualquier precio, ya que el principio sobre el cual las enfermeras han sido educadas es el código moral que cree en respeto hacia la vida humana. La preservación de la vida es un mandamiento moral absoluto y por lo cual, segun Kant, la enfermera debe forzar al paciente ha ser tratado.

\section{Qué dice la "Halacha" (Parte legal de la literatura judía tradicional)}

Curar es un deber; sin embargo, al doctor se le da permiso de curar (BIBLIA) ${ }^{13}$. Por naturaleza, el principal propósito del ser humano es el de vivir y está obligado a hacer cualquier cosa en orden de seguir viviendo hasta que el Señor decida lo contrario. La cuestión de salvar vida, uno de los más importantes tópicos en la "Torá"; es superior a la mayoría de las otras reglas. La "Halacha" obliga a sus concomitantes a forzar la alimentación en sus pacientes; sí no lo harán de esta forma serán concebidos como pecadores (LEVITICO, 13) ${ }^{14}$. Además, cualquier persona que acorta la vida de outro es considerado como un asesino (PROSCHT, 1992) ${ }^{7}$. Desde el punto de vista del judaísmo la discusión está llena de complejos dialectos. Los valores básicos de santidad de la vida y de su preservación a todo costo están en conflicto con cuestiones actuales que contrastan la percepción de los derechos humanos. La "Torá" enfatiza el veto de acortar la vida humana y recalca el deber de realizar todo lo necesario para salvarla.

\section{EL PAPEL DE LAS ENFERMERAS GERIÁTRICAS}

Las enfermeras son parte de la comunidad, el medio ambiente y la sociedad.
Sus obligaciones tienen características normativas y de comportamiento que dictan reglas y política local. Mientras en el estado de Israel no exista una explícita ley, la cual permita suspender la alimentación del paciente, la enfermera deberá encontrar la más apropiada clase de tratamiento.

Cada institución tiene un determinado marco de trabajo sobre el cual define su política y trata el problema de alimentación de los pacientes ancianos dementes. La función de las enfermeras geriátricas es la de involucrar a la familia del paciente en la administración del tratamiento y así reducir mutuos conflictos.

El proceso asistencial es una de las más importantes herramientas en el trabajo de las enfermeras. Es esta la herramienta que permite planear tratamientos, a corto y largo plazo, consecuente y libremente.

$\mathrm{Al}$ diagnosticar la demencia en el justo momento nos permite dar respuestas apropiadas; especialmente en casos en los cuales el proceso está acompañado por una continua evaluación, permitiendo cambios en el programa del tratamiento, antes de tomar decisiones acerca de nuevas intervenciones.

La definición del problema, puntos de vista y la relación entre "el uno mismo" y la profesión, permiten a la enfermera a considerar varias opciones de tratamiento, examinar uno por uno y finalmente decidir cual de ellos es el más apropiado.

Esta elección es realizada con la cooperación de la familia para lograr entendimiento, menos dificultades y el sentimiento de compañerismo. Tal intervención se va desarrollando por etapas.

La habilidad profesional de la enfermería, mientras esta manejando el problema de alimentación forzada, y el de su relación con la familia contribuyen de una gran forma al éxito del tratamiento en pacientes dementes. La enfermera esta capacitada para crear una atmósfera psicosocial, llevar a cabo el tratamiento físico y aconsejar a la familia en todos los asuntos acerca de la rehabilitación del paciente.

La familia puede entrar en un estado de frustación, crisis o de incapacidad; como consecuencia, la relación con la familia se inestabiliza. En orden de evaluar la calidad de tratamiento, no hay duba de que debemos guiar a la familia de como prevenir luchas y resolver conflictos; debemos aplicar el método de "la casa abierta".

Aunque las enfermeras siempre vacilan acerca de los métodos de tratamiento que deben aplicar, la enfermera especializada en geriatría debe colocar su papel de aliviar el sufrimiento del paciente en la cima de sus prioridades; ellas tratan todo el tiempo de mejorar la condición y de aliviar el sufrimiento de estos pacientes. Estos esfuerzos son realizados expresando empatía y tolerancia. De este forma, la enfermera especializada en geriatría es el propotipo de la enfermera clínica, lo cual significa:

un participante directo - desarrollando programas de tratamiento para los pacientes geriátricos. 
un coordinador: coordina el tratamiento en el instituto con los otros disciplinas, combinandolos todos juntos.

un consejero - aconseja como promover ciertos programas de tratamiento.

un educador - guía al paciente, a su familia y al personal de tratamiento usando principios de manejo del dolor.

un investigador - realiza investigación en el campo de tratamiento de pacientes geriátricos. La enfermera evalua los hallazgos de la investigación y los combina con los métodos prácticos.

un director estratégico - combina nuevos tratamientos tecnológicos y un apropiado plan de tratamiento. Existe la posibilidad de si, dedicando una determinada enfermera a un determinado paciente, podemos establecer mejores relaciones entre ellos (escuchando la misma voz, sintiendo el mismo tacto, etc); por lo tanto, se reducen los sentimientos de estar tratando con un "proyecto de alimentación" (MERCHANT, 1985) ${ }^{15}$.

como un participante directo - observa a estudiantes de enfermería mientras tratan a los pacientes; la enfermera evalua su desempeño y los guía.

Las enfermeras son las que traducen sus calificaciones en acciones. Ellas proporcionan tratamiento asistencial de alta calidad, tomando en consideración otros factores, tales como el paciente, la enfermedad y la familia.

El conocimiento de las enfermeras es muy vasto y sólido lo que constituye la base para su intervención y evaluación y les permite tener una vasta perspectiva para proveer el apropiado tratamiento.

La conferencia se convocó con la participación de un doctor, un rábino, un abogado, una enfermera, el personal médico del instituto, el director y la junta directiva.

\section{CONCLUSIONES}

01. Cada caso sera analizado por un cuerpo interdisciplinario.

02. Las decisiones seran tomadas tanto por el personal médico como por el personal asistencial.

03. Los procedimientos concernientes al comportamiento del personal en estos casos, sera explicitamente notificado por escrito.

04. Es recomendable que la opinión del paciente sea documentada en su expediente, antes de cualquier cambio en su estado mental.

05. Varias conferencias se convocarán cada año, para analizar casos asistenciales complejos. Estas conferencias incluirán personal interdisciplinario de todos los institutos geriátricos en Israel.

06. Talleres educativos para personal relacionado con problemas de alimentación serán sostenidos.

\section{RESUMEN}

Asistencia es un ciencia que describe el proceso cognitivo y de actuacion del tratamiento de pacientes ancianos.

La ciencia asistencial trata de mejorar/preservar la calidad de vida de los ancianos; tambien trata de crear condiciones que permitiran "una muerte digna".

La importancia de la asistencia aumenta cuando el paciente anciano esta confuso, en estado de demencia definitiva.

La tendencia de las enfermeras es la de aumentar la estimulación externa proporcionada al paciente, para fortalecer el contacto con el/ella y disminuir sus reacciones violentas, especialmente en los casos de alimentación forzada.

\section{FRUITS OF TECHNOLOGY - SHOULD WE FEED AN OLD PATIENT AT ALL COSTS?}

The purpose of this article is to analyze questions on a complex ethic, medical and caring dilemma about reasons to continue or suspend compelled alimentation in really old patients carrying progressive acute dementia. Traditionally, the principle of life holiness supports the idea that life should be extended at all costs and the decision to continue feeding the patients is not questioned. However, revolutionary ideas are arising about attitudes, concepts and value of treatment, conducting an urgent need to discuss this problem deeply. Until when should we compel demented patients to eat, as they do not accept food and have to be fed through a catheter or intraveined alimentation, tying back their hands or using other measures? This discussion occurred in a geriatric institution at Israel, when a doctor's order was not accepted by nurses, as they had a different way of seeing the problem. A national conference on this theme was organized with the attendance of doctors, nurses, social workers and psychologists.

KEY WORDS: elderly people, compelled alimentation, geriatric nurse

\section{REFERENCIAS BIBLIOGRAFICAS}

01. GOMPER, M. La edad y la pérdida de la productividad. Essay. Asociación Israelí de Gerantología, 1971.
02. HAZAN, H. Los ancianos como fenómeno social. Ministerio de Defensa, 1984. p. 35-39.

03. MOTLIS, H. Muchas caras hacia los ancianos. Kibbutz Meuchad. 4, 1989. p. 108-118. 
04. SCHOSTAK, Z. Ética Judía de resucitación, nutrición artificial e hidratación de los ancianos. Revista de Ética Médica, v. 20, p. 93-100, 1994.

05. JACOBOVITCH, E. Los judíos y la medicina. La nueva época, v. 36, p. 57, julio 1989.

06. ZONGEBLIC, M. Dilemas de los ancianos. Los judíos y la medicina. La nueva época. v. 36, p. 5-7, 1989.

07. PROSCHT, G. Los derechos humanos en el judaísmo. Jerusalém, Instituto Sanhedrin, 1992. cap. 1, p. 19-87.

08. NORBERG, A.; HIRSCHFELD, M. Alimentación de pacientes severamente dementes en instituciones: entrevista con proporcionadores de cuidado en Israel. J.Adv.Nurs, v. 12, p. 551$557,1987$.
09. DRESSER. Asuntos éticos en enfermería. v. 33, n. 11, p. 790-794, 1985.

10. LYNN et al. Asuntos éticos en el cuidado de ancianos residentes en institutos asistenciales. Ética Biomedica, v. 13, n. 2, 1986.

11. NORBERG, A. et al. Razonamiento ético acerca de la alimentación de pacientes severamente dementes: una perspectiva internacional. Ética Asistencial, v. 1, n. 1, p. 3-15, march. 1994.

12. MILMOE, P.; CARRICK, M. Continuar o retirar la nutrición o hidratación. Centro de literatura bioética, nov., 1986.

13. BIBLIA. Exodo. Bíblia sagrada. Capítulos 29,19.

14. BIBLIA. Levítico. Bíblia sagrada. Capítulo 13.

15. MERCHANT. Porqué repartir tareas?. Pract. Asistencial., v. 2, p. 67, 1985. 